FORMATION Formation emploi

Revue française de sciences sociales

133 | Janvier-Mars 2016

Heurs et malheurs de l'apprentissage en Suisse

\title{
Les réseaux d'entreprises formatrices : une nouvelle forme d'apprentissage en Suisse
}

Training networks: A new form of apprenticeship in Switzerland

Lehrbetriebsverbünde - eine neue Form von Berufslehre in der Schweiz

Las redes de empresas formadoras : una nueva forma de aprendizaje en Suiza

Regula Julia Leemann, Sandra Da Rin et Christian Imdorf

\section{(2) OpenEdition}

Journals

Édition électronique

URL : http://journals.openedition.org/formationemploi/4666

DOI : 10.4000/formationemploi.4666

ISSN : 2107-0946

Éditeur

La Documentation française

Édition imprimée

Date de publication : 20 avril 2016

Pagination : 139-156

ISSN : 0759-6340

\section{Référence électronique}

Regula Julia Leemann, Sandra Da Rin et Christian Imdorf, « Les réseaux d'entreprises formatrices: une nouvelle forme d'apprentissage en Suisse », Formation emploi [En ligne], 133 | Janvier-Mars 2016, mis en ligne le 21 avril 2018, consulté le 30 octobre 2020. URL : http://journals.openedition.org/ formationemploi/4666; DOI : https://doi.org/10.4000/formationemploi.4666 


\title{
Les réseaux d'entreprises formatrices: une nouvelle forme d'apprentissage en Suisse
}

\author{
Regula JuLia LeEmanN \\ Prof. Dr., Chaire de sociologie de l'éducation \\ Haute école pédagogique de la Suisse du Nord-Ouest, Bâle \\ SANDRA DA RIN \\ Lic. phil., Sociologue \\ Ecole de formation professionnelle, supérieure et continue de Berne \\ CHRISTIAN IMDORF \\ Prof. Dr., Sociologue \\ Institut de sociologie, université de Berne
}

Résumé

\begin{abstract}
Les réseaux d'entreprises formatrices : une nouvelle forme d'apprentissage en Suisse.

Les réseaux d'entreprises formatrices constituent un modèle du système de formation professionnelle en alternance suisse. Petites et moyennes entreprises peuvent ainsi mutualiser la formation des apprentis. Quelles raisons poussent les entreprises à participer à ce nouveau type d'organisation ? Quels conflits et tensions naissent au sein de ces réseaux ? Les analyses s'appuient sur quatre cas de réseaux et sur la théorie de l'économie des conventions. Ces réseaux naissent d'une pluralité de motifs de participation, source d'insatisfaction dans les entreprises et de conflits dans les réseaux tout au long du parcours de formation.
\end{abstract}

Mots clés : enseignement technique-professionnel, apprentissage, réseau d'entreprises, organisation qualifiante, PME-PMI, économie des conventions, organisation du travail, Suisse

Abstract

Training networks: A new form of apprenticeship in Switzerland

Training networks are a model of vocational training in the dual VET system in Switzerland. Small and medium-sized enterprises can train apprentices jointly. Drawing on four case studies of training networks in Switzerland and on the theory of the economics of conventions, two questions are treated: What are the motives of training 
companies for participation in a training network? Which conflicts and tensions arise from the joint training of apprentices? The results demonstrate the plurality of motifs for participation in a training network, resulting in dissatisfaction of the training companies and ongoing conflicts in the network over the course of the training.

Keywords : technical \& vocational education, apprenticeship, network of companies, skilling organisation, SME, economy of conventions, work organisation, Switzerland

Journal of Economic Literature: L 14, M 51, M 53

Traduction : Auteur-e-s.

En raison des évolutions technologiques, économiques et sociales, le système de formation professionnelle en alternance est appelé à adapter son mode d'organisation pour répondre à ses missions de qualification et d'intégration. Dans ce contexte sont nés, au cours des deux dernières décennies, en Suisse (mais aussi dans d'autres pays), les réseaux d'entreprises formatrices (REF) dans lesquels des petites et moyennes entreprises (PME) mutualisent la formation des apprentis. Ces derniers tournent entre les différentes entreprises. Une organisation principale est chargée de recruter les apprentis et d'organiser la formation.

Ce dispositif soulève deux questions : Quelles sont les raisons qui poussent les entreprises à participer à ce nouveau type d'organisation? Quels sont les conflits et les tensions qui naissent au sein du REF ?

Dans un premier temps, nous présenterons le modèle REF mis en place en Suisse et les résultats d'une évaluation de l'Office fédéral suisse de la formation professionnelle et de la technologie (OFFT). Ils fournissent un premier aperçu des initiatives et des motifs de création, des objectifs, des avantages et inconvénients des REF (OFFT, 2008). Ensuite, nous exposerons les résultats d'une enquête empirique concernant quatre études de cas de REF afin d'identifier les motifs de la participation des entreprises à ces réseaux. Enfin, nous présenterons plus spécifiquement les conflits et tensions propres au REF. A cet effet, nous mobiliserons le cadre théorique de l'économie des conventions.

\section{Les réseaux d'entreprises formatrices peinent à se développer}

En pratique, divers modèles de système de réseaux d'entreprises formatrices (REF) existent (BMBF 2011). Celui étudié ici en Suisse est soutenu par la Confédération depuis la fin des années 1990 , par le biais de la diffusion d'informations ${ }^{1}$ et d'aides financières ${ }^{2}$.

1. http://www.lbv.berufsbildung.ch/dyn/3545.aspx, 12.6.2015.

2. http://www.sbfi.admin.ch/berufsbildung/01545/index.html?lang=fr, 12.6.2015. 
Comparé à l'apprentissage " classique ", ce modèle se distingue par le fait que les apprentis changent d'entreprise formatrice tout au long de leur période d'apprentissage. ${ }^{3}$ Selon les métiers, cela a lieu tous les six à douze mois.

Une organisation principale, interentreprises, est responsable de la mise en place et du fonctionnement du réseau. Elle choisit les apprentis, conclut avec eux le contrat d'apprentissage et leur attribue les entreprises formatrices par rotation. L'organisation principale soutient les entreprises dans leurs objectifs de formation par le biais de coaching et en assurant la communication entre les entreprises. Ces dernières la rémunèrent pour ces services. Un contrat écrit stipule les attributions, prestations et responsabilités respectives des entreprises et de l'organisation principale.

Deux personnes accompagnent les apprentis. L'une, issue de l'organisation principale, est le maître d'apprentissage officiel et, à ce titre, il est le responsable juridique de la formation de l'apprenti ; l'autre, au sein des entreprises formatrices, est un formateur pratique responsable de la formation technique des apprentis. Les changements d'entreprise exigent des jeunes une grande flexibilité et une certaine ouverture d'esprit ; ils doivent en effet s'adapter à plusieurs reprises et rapidement à des champs d'activité encore inconnus, à de nouvelles équipes de travail et à une clientèle différente (Leemann et Sagelsdorff, 2014). En revanche, ce système promeut les compétences professionnelles et les compétences-clés (Leemann et Birr, 2015).

En matière de politique de formation professionnelle, les REF visent divers objectifs : permettre une qualification plus large des apprentis, créer de nouvelles places d'apprentissage, garantir un recrutement et un accompagnement professionnel conformes aux objectifs de formation, favoriser l'intégration des jeunes socialement défavorisés et offrir des places d'apprentissage attractives (Walther et Renold, 2005 ; Hoeckel et al., 2009, p. 40 ; Drinkhut et Schlottau, 2003, p. 31).

En Suisse, une évaluation des REF, réalisée en 2008 par l'OFFT, présente les principaux résultats du point de vue des organisations principales et des entreprises membres des REF questionnés ; les questions portaient notamment sur les initiatives et les motifs de création de ce dispositif, les objectifs de formation et les avantages et inconvénients (OFFT, 2008).

La création de réseaux est à l'initiative, d'une part, d'acteurs économiques : entreprises (32\%) et organisations du monde du travail, comme par exemple les associations professionnelles (12\%) ; d'autre part, d'acteurs gouvernementaux : organisations de services publics $(16 \%)$ ou à but non lucratif $(24 \%)$. Dans la plupart des cas, l'organisation principale se consacre uniquement à la formation des apprentis. Elle est organisée en association dans les trois quarts des cas (OFFT, 2008, p. 8).

3. Voir « Réseaux d'entreprises formatrices" (http://www.berufsbildung.ch/dyn/bin/3545-15759-1-am19. pdf, 12.6.2015). 
Pour la majorité des organisations principales ( $80 \%)$, la création ou la garantie de places d'apprentissage a été décisive dans la mise en place d'un réseau d'entreprises formatrices. Lobjectif visant à augmenter la qualité de la formation est a contrario peu évoqué (36\%). L'insertion de jeunes ayant un déficit de formation ou des problèmes d'insertion a été une source de motivation pour un cinquième des entreprises (OFFT, 2008, p. 9). Ces motifs généraux peuvent être comparés à des raisons spécifiques de participer à une formation en réseau (OFFT, 2008, p. 27) :

- L'impossibilité, pour les entreprises, de couvrir tout l'éventail de la formation et de former seules (environ les trois-quarts des entreprises);

- Le soutien reçu au cours de la formation et la possibilité de déléguer une partie des responsabilités à l'organisation principale, par exemple : accompagnement des apprentis, recrutement, gestion de salaire, formation complémentaire des apprentis, cours de rattrapage (OFFT, 2008, p. 21) (environ les deux tiers des entreprises);

- Le manque d'expérience en matière de formation des apprentis et le besoin d'accompagnement (environ un tiers des entreprises);

- La possibilité de ne pas s'engager sur plusieurs années (environ un quart des entreprises).

Ainsi, la formation mutualisée des apprentis en réseau et le soutien apporté par l'organisation principale sont au centre des motivations des entreprises. A l'inverse, les organisations principales poursuivent des buts supérieurs axés sur les missions de qualification et d'insertion de la formation professionnelle.

Pour les entreprises, les avantages sont (par ordre d'importance) : « Flexibilité des apprentis par la rotation; Formation dans plusieurs entreprises pour accroître la qualité de la formation; Possibilité, pour l'entreprise, de se concentrer sur sa compétence principale; Transfert de connaissances entre les entreprises membres; Connaissance exacte des coûts liés à l'activité de formation ; Plus large choix pour le recrutement des apprentis" (OFFT, 2008, 28).

Néanmoins, les entreprises mentionnent aussi des inconvénients potentiels et les plus souvent cités sont : "Effort pour initier sans cesse de nouveaux apprentis; Durée de séjour trop courte pour pouvoir profiter des apprentis; Difficulté pour les apprentis à sintégrer en raison de la brièveté de leur séjour; Risque de divulgation des secrets professionnels de l'entreprise à cause de la rotation; Surmenage des apprentis à cause du changement d'entreprises de formation; Paiement par les entreprises de services dont elles n'auraient pas besoin; Concurrence des entreprises membres lors de l'engagement des jeunes formés; Ingérence de l'entreprise I de l'organisation principale dans la formation ".

Malheureusement, il n'existe pas de statistiques concernant le nombre de places d'apprentissage ou de REF. Sur la base de l'évaluation de l'OFFT (2008) et d'un inventaire que nous avons réalisé dans le cadre d'une recherche sur Internet, nous estimons que le nombre de places d'apprentissage REF représente $3 \%$ de toutes les places d'apprentissage disponibles dans le système en alternance. 
Bien que ce dispositif représente un modèle prometteur pour les entreprises, les apprentis et pour la formation professionnelle en tant qu'institution, le nombre des REF peine à se développer. Ce système de gestion en réseau est complexe et ambitieux. Il concerne en effet plusieurs entreprises aux intérêts pluriels, comporte un système de rotation des apprentis et deux acteurs responsables de l'accompagnement, autant d'éléments susceptibles d'engendrer des difficultés.

Cela conduit à deux interrogations que nous aimerions aborder ci-après, en nous appuyant sur une approche théorique et une enquête empirique : quelles sont les raisons qui incitent les entreprises à participer à ce nouveau type de modèle organisationnel ? Quels sont les conflits et les tensions qui naissent au sein du REF ?

\section{Les raisons plurielles de l'engagement des entreprises}

La plupart des études qui analysent les motifs conduisant les entreprises individuelles à investir dans la formation professionnelle initiale en modèle traditionnel ont une approche coûts-bénéfices (pour la Suisse, Strupler et Wolter, 2012 ; pour l'Allemagne, Schönfeld et al., 2010). On peut y distinguer deux raisons clés : celle de la production et celle de l'investissement (Wenzelmann, 2012, p. 127).

Les raisons liées à la production mettent l'accent sur la productivité des apprentis. Les entreprises proposent une formation pour avoir accès à une main-d'œuvre bon marché et profiter ainsi rapidement, après le début de la formation et pour la durée de l'apprentissage, des capacités de travail des jeunes.

Les raisons liées à l'investissement visent à assurer, à long terme, la relève professionnelle de l'entreprise et la productivité des apprentis après la formation. L'entreprise a donc tout intérêt à maintenir un niveau élevé de formation et d'investir en ce sens. Pour éviter les mauvais investissements, il est nécessaire que les apprentis restent dans l'entreprise après la formation, ce qui implique de transmettre un certain attachement à l'entreprise.

Au-delà de ces deux motivations, la littérature en évoque d'autres. Selon le "motif de réputation "(Wenzelmann, Ibid.), les entreprises espèrent que leur engagement dans la formation leur vaudra l'estime de leurs clients, de leurs employés et de leurs autres partenaires commerciaux. Pour d'autres, la formation des apprentis s'inscrit dans la tradition des entreprises (Wenzelmann et al., 2009).

Pour tenter de comprendre les raisons de la participation d'entreprises à un réseau et des conflits et tensions qui naissent au sein du REF, la théorie de l'économie des conventions offre des perspectives riches. ${ }^{4} C^{\prime}$ est une approche transdisciplinaire qui s'inscrit dans la

4. Pour une brève introduction, voir Eymard-Duvernay, 2008. 
nouvelle sociologie pragmatique française (Boltanski et Thévenot, 1991 ; Boltanski et Chiapello, 1999 ; Diaz-Bone et Thévenot, 2010). En analysant des organisations, cette théorie formule l'hypothèse d'une coexistence de logiques d'action concurrentes et conflictuelles, ainsi que celles de conflits et de zones de tension en découlant (Jagd, 2011).

Dans cette perspective, les acteurs effectuent leurs évaluations et prennent leurs décisions en s'appuyant sur diverses conventions, constituées au fil du temps, qui formatent la perception cognitive, coordonnent les actions et légitiment les jugements. Ces conventions s'inscrivent dans des "mondes de la justification" (Boltanski et Thévenot, Ibid.). Pour notre étude, nous retiendrons les mondes industriel, civique, par projets, domestique, de l'opinion et marchand (voir Tableau 1). La détermination d'un monde de justification s'appuie sur leur "logique d'action". Chacun de ces mondes se caractérise par la " grandeur" ou " la qualité" des choses, des personnes et des processus au regard du bien commun, "qui résulte d'un accord entre les personnes sur la finalité commune de leur action" (Eymard-Duvernay, 2008, p. 58).

Tableau 1: Mondes de la justification et logiques d'action (conventions)

\begin{tabular}{|c|c|c|c|}
\hline & $\begin{array}{l}\text { Logique d'action } \\
\text { Qualité (grandeur) }\end{array}$ & $\begin{array}{l}\text { Entreprises : Motifs de } \\
\text { participation }\end{array}$ & $\begin{array}{c}\text { Entreprises : conflits et } \\
\text { tensions (exemples) }\end{array}$ \\
\hline $\begin{array}{l}\text { Monde } \\
\text { industriel }\end{array}$ & $\begin{array}{l}\text { Efficacité productive, expertise, } \\
\text { maîtrise }\end{array}$ & $\begin{array}{l}\text { Investissement pour le futur de l'entreprise/ } \\
\text { de la branche : Former une relève (plus) } \\
\text { qualifiée. Accroître la qualité de la formation }\end{array}$ & $\begin{array}{l}\text { Critiques du monde industriel } \\
\text { envers le monde marchand : } \\
\text { Concurrence entre les entreprises } \\
\text { qui empêche d'attirer suffisamment } \\
\text { d'apprentis diplômés afin de les } \\
\text { employer }\end{array}$ \\
\hline Monde civique & $\begin{array}{l}\text { Solidarité, priorité au collectif, } \\
\text { égalité, intégration sociale }\end{array}$ & $\begin{array}{l}\text { Intégration professionnelle des jeunes } \\
\text { (socialement défavorisés). Leur permettre } \\
\text { d'accéder à une formation professionnelle }\end{array}$ & --- \\
\hline $\begin{array}{l}\text { Monde par } \\
\text { projets }\end{array}$ & $\begin{array}{c}\text { Réseau dynamique et chan- } \\
\text { geant, activité, flexibilité, } \\
\text { mobilité }\end{array}$ & $\begin{array}{c}\text { Acquisition des compétences clés chez les } \\
\text { apprentis (flexibilité, aptitude à la mobi- } \\
\text { lité) par le système de rotation. Maitriser } \\
\text { les exigences d'un monde du travail en } \\
\text { mutation }\end{array}$ & --- \\
\hline
\end{tabular}




\begin{tabular}{|c|c|c|c|}
\hline Monde inspiré & $\begin{array}{l}\text { Inspiration, passion, vocation, } \\
\text { motivation intrinsèque }\end{array}$ & $\begin{array}{l}\text { Proposer une formation variée et passion- } \\
\text { nante, afin d'augmenter le potentiel de } \\
\text { recrutement et de prévenir les ruptures de } \\
\text { contrat durant l'apprentissage }\end{array}$ & --- \\
\hline $\begin{array}{l}\text { Monde de } \\
\text { l'opinion }\end{array}$ & $\begin{array}{l}\text { Réputation, renommée, visibi- } \\
\text { lité, popularité }\end{array}$ & $\begin{array}{l}\text { Attirer des jeunes qualifiés par une image } \\
\text { attractive du réseau, par des marques fortes } \\
\text { (entreprises bien connues) }\end{array}$ & $\begin{array}{l}\text { Critiques du monde de l'opinion } \\
\text { envers le monde civique : } \\
\text { Mauvaise image du réseau qui } \\
\text { donne sa chance à chacun }\end{array}$ \\
\hline $\begin{array}{l}\text { Monde } \\
\text { marchand }\end{array}$ & $\begin{array}{l}\text { Prix, gain, concurrence, bon } \\
\text { rapport qualité-prix }\end{array}$ & Utilisation flexible de personnel bon marché & $\begin{array}{l}\text { Critiques du monde marchand } \\
\text { envers le monde par projets : } \\
\text { La rotation aboutit à des jeunes }\end{array}$ \\
\hline $\begin{array}{l}\text { Monde } \\
\text { domestique }\end{array}$ & $\begin{array}{l}\text { Hiérarchie, autorité, traditions, } \\
\text { relations interpersonnelles }\end{array}$ & --- & $\begin{array}{l}\text { Critiques du monde domestique } \\
\text { envers le monde par projets: } \\
\text { La rotation conduit à la perte d'au- } \\
\text { torité et d'influence de l'entreprise }\end{array}$ \\
\hline
\end{tabular}

Source : Représentation élaborée par les auteurs et s'inspirant des travaux de Boltanski et Thévenot (1991), et de Boltanski et Chiapello (1999), complétée sur la base des résultats.

Dans cette perspective, les situations sociales comme la participation de différentes entreprises à une formation commune d'apprentis font converger différents mondes de justification. Les acteurs agissent dans une situation selon la logique d'action ; ils justifient leurs attentes et décisions en fonction de cette logique et dénoncent les autres logiques mises en jeu par les partenaires du réseau.

Afin de demeurer capacité d'agir et de ne pas mettre en danger la formation, les acteurs sont in fine obligés de faire des compromis entre les mondes de justification. Ces compromis visent à gérer les conflits potentiels entre logiques d'action divergentes.

Quatre réseaux ont été étudiés dans une approche qualitative (voir encadré 1 et tableau 2). Ils ont été choisis afin de parvenir - dans le contexte de la théorie sousjacent - à des résultats contrastants (réplications théoriques dans le sens de Yin, 2009, p. 54).

Outre la taille qui détermine les exigences de la gestion d'un REF, les critères de sélection avaient trait à l'historique de la création et à l'organisation principale ayant la responsabilité institutionnelle du REF. Quant aux REF à l'initiative d'entreprises et d'associations professionnelles (acteurs économiques), selon nous, le but prioritaire est d'assurer une relève qualifiée dans les entreprises ou dans un secteur d'activité. S'agissant des REF à l'initiative des communes et organisations caritatives et non lucratives (acteurs gouvernementaux), l'objectif est plutôt de répondre à des problèmes sociaux (entre autres, le manque de places d'apprentissage, la discrimination subie par 
les jeunes lors de la recherche d'une place d'apprentissage). Cela est possible grâce à la création de nouvelles places d'apprentissage et au travail professionnel de l'organisation principale (sélection rigoureuse : examen des dossiers de candidature, entretiens, stages...) (OFFT, 2008, p. 9).

Deux cas représentent des REF créés par des acteurs économiques. Il s'agit du « réseau de l'expédition » et du " réseau des transports publics ». Les deux autres retenus - le « réseau région » et le "réseau intégration »- ont été créés par des acteurs gouvernementaux. ${ }^{5}$

\section{Encadré 1 : Méthodes}

\section{Etude}

Les données sont issues du projet de recherche effectué grâce aux subventions du Fonds National Suisse (FNS) : "Les réseaux d'entreprises formatrices dans la pratique ». Chef de projet : Regula Julia Leemann et Christian Imdorf. Durée de la recherche : 6/2011-11/2014 (FNS-13DPD3_134855). Collaboratrices : Lorraine Birr, Sandra Da Rin, Rebekka Sagelsdorff, Nicolette Seiterle.

\section{Collecte des données}

Trois unités d'analyse ont été prises en compte lors de la collecte de données : l'organisation principale (entretiens d'experts avec directeur et maîtres d'apprentissage), les entreprises formatrices (entretiens d'experts avec des responsables de la formation : conseiller de formation, formateur pratique) et les apprentis (entretiens guidés). Les analyses de l'article s'appuient sur des entretiens avec les responsables de la formation (sept-neuf entreprises par REF - réseau d'entreprises formatrices) et sur la documentation fournie par les REF (rapports de gestion, concepts de formation).

Thèmes utilisés dans le cadre des entretiens avec les responsables des entreprises formatrices pour la formation des apprentis :

- Raisons de la participation ;

- Expériences positives/problématiques et justifications des évaluations positives/ problématiques (coopération entre entreprises, soutien de la part de l'organisation principale, recrutement, principe de rotation, apprentis) ;

- But de la formation d'apprentis ;

- Développements futurs.

Méthodes utilisées pour la conduite des entretiens et pour le traitement du matériau recueilli

Partant de la théorie de la justification fondée sur la théorie de l'action, mais orientée vers le pragmatisme (Diaz-Bone, 2011, p. 48), la conduite des entretiens et le traitement des données visaient à recueillir des justifications de décisions prises et des expériences faites. II s'agissait de reconstituer les mondes de justification ainsi que les conflits et les compromis entre ces eux.

5. Les noms des réseaux sont choisis pour la clarté de la présentation des résultats, puisqu’ils font déjà apparaître certains enjeux. 
Tableau 2 : Quatre cas étudiés issus des réseaux d'entreprises formatrices

\begin{tabular}{|c|c|c|}
\hline & « Réseau de l'expédition » & « Réseau des transports publics» \\
\hline $\begin{array}{l}\text { Initiative à l'origine du } \\
\text { projet }\end{array}$ & Associations d'entreprises et de secteurs d'activité & $\begin{array}{l}\text { Deux entreprises et une association de secteurs } \\
\text { d'activités }\end{array}$ \\
\hline Taille & $\begin{array}{l}\quad \approx 35 \text { entreprises membres } \\
\quad \approx 40 \text { apprentis } \\
4 \text { employés (organisation principale) }\end{array}$ & $\begin{array}{l}\approx 60 \text { entreprises membres en notant qu'une } \\
\text { entreprise couvre } 80 \% \text { des places de formation } \\
\text { (entreprise fédérale). } \\
\approx 1800 \text { apprentis } \\
\approx 280 \text { employés (organisation principale) }\end{array}$ \\
\hline $\begin{array}{l}\text { Métiers proposés à } \\
\text { l'apprentissage }\end{array}$ & $\begin{array}{c}\text { Une profession } \\
\text { Employé de commerce en logistique internationale de } \\
\text { l'expédition }\end{array}$ & $\begin{array}{l}\qquad \approx 25 \text { professions } \\
\text { La profession la plus représentée : Employé-e de } \\
\text { commerce en transports publics }\end{array}$ \\
\hline & " Réseau région » & " Réseau intégration » \\
\hline $\begin{array}{l}\text { Initiative à l'origine du } \\
\text { projet }\end{array}$ & $\begin{array}{l}\text { Office cantonal de formation et de l'orientation } \\
\text { professionnelle }\end{array}$ & $\begin{array}{l}\text { Centre d'orientation scolaire et professionnelle } \\
\text { d'une grande ville }\end{array}$ \\
\hline Taille & $\begin{array}{l}\quad \approx 15 \text { entreprises membres } \\
\quad \approx 55 \text { apprentis } \\
4 \text { employés (organisation principale) }\end{array}$ & $\begin{array}{c}\approx 150 \text { entreprises membres } \\
\approx 220 \text { apprentis } \\
\approx 20 \text { employés (organisation principale) }\end{array}$ \\
\hline $\begin{array}{l}\text { Métiers proposés à } \\
\text { l'apprentissage }\end{array}$ & $\begin{array}{c}7 \text { professions } \\
\text { Employé de commerce et métiers techniques (par } \\
\text { exemple Polymécanicien/ne) }\end{array}$ & $\begin{array}{l}14 \text { professions dans quatre secteurs d'activité } \\
\text { (par exemple employé de commerce, assistant/e } \\
\text { socio-éducatif/ve) }\end{array}$ \\
\hline
\end{tabular}

\section{La participation des entreprises : entre investissement et engagement civique}

Pourquoi des entreprises participent-elles à la formation professionnelle en réseau, alors que ce dispositif est plus complexe que l'apprentissage " traditionnel »? Deux principales raisons apparaissent dans le discours des entreprises en REF : premièrement, assurer sur le long terme la formation de personnel disposant d'une bonne qualification pour sa propre entreprise, pour une filière ou pour un secteur d'activité précis ; deuxièmement, favoriser l'intégration professionnelle des jeunes (socialement défavorisés).

\subsection{Accroître la main d'œuvre qualifiée et favoriser l'insertion des jeunes socialement défavorisés ...}

La première raison principale est liée à l'investissement : il n'est possible qu'avec un réseau qui permet de devenir plus professionnel. En raison de leur spécialisation, certaines entreprises ne peuvent pas proposer tout l'éventail de la formation qui leur est nécessaire ; de ce fait, elles sont dépendantes d'une collaboration avec d'autres entreprises. 
La rotation entre les entreprises permet aux jeunes de découvrir un large éventail de champs d'activité. C'est parfois indispensable, à l'instar du métier d'assistant/e socio-éducatif/ve, (variante généraliste) dont le curriculum de formation prévoit respectivement une année de formation pour l'accompagnement des personnes âgées, une auprès des personnes handicapées et une autre consacrée aux enfants. Ce modèle de formation ne peut être mis en place que si une maison de retraite, un centre pour handicapés et une crèche sont co-responsables et co-organisateurs de la formation.

De nombreuses entreprises en réseau insistent également sur l'importance des compétences professionnelles de l'organisation principale et indiquent qu'elles ne sont pas expertes en formation. C'est pourquoi certaines entreprises délèguent la formation professionnelle à l'organisation principale. Grâce à la mise en commun de ressources, cette dernière peut recruter les candidats/tes à l'apprentissage de manière professionnelle en procédant par étapes lors de la procédure de sélection (présélection des dossiers de candidature, entretiens, stage court dans une entreprise), ce qui permet d'en décharger les entreprises. Ces dernières n'ont plus besoin d'effectuer seules ce choix «risqué» et chronophage (Imdorf et Leemann, 2012 ; Imdorf et Seiterle, 2015). En outre, l'organisation principale aide les entreprises dans l'accompagnement des apprentis, surtout s'agissant des problèmes scolaires et personnels des jeunes. De plus, les petites entreprises sont souvent dépassées par les changements incessants en matière de réglementation de la formation professionnelle ; l'organisation principale les aide alors en proposant des formations continues destinées aux formateurs pratiques des entreprises.

Ces différents aspects de la construction sur le long terme d'un groupe de personnel qualifié renvoient à une logique dominante de la convention industrielle qui place la formation professionnelle en son centre et vise à former, sur le long terme, de la main-d'œuvre qualifiée (voir tableau 1).

Le second motif principal de l'investissement des entreprises dans un réseau est l'intégration professionnelle des jeunes en général et en particulier des jeunes socialement défavorisés. Ce motif vise, d'une part, à permettre à des jeunes d'accéder à une formation professionnelle et, d'autre part, à mieux les préparer aux exigences du monde du travail. Quelques entreprises déclarent aussi qu'elles souhaitent donner une chance à des jeunes en difficulté sur le marché des places d'apprentissage, des jeunes socialement discriminés ou de faible niveau scolaire. Tous ces arguments, qui s'inspirent de l'égalité des chances, de l'intégration sociale et professionnelle et de la garantie du diplôme de fin de formation professionnelle, s'appuient sur les justifications du monde civique.

Ces deux raisons clés structurent la participation de toutes les entreprises au réseau. Néanmoins, toutes les entreprises ne s'appuient pas de la même façon sur ces deux mondes de justification. Les discours issus du monde civique se retrouvent plutôt chez les employeurs du secteur public, comme par exemple dans les chemins de fers du « réseau des transports publics", dont les principaux actionnaires sont des cantons et la Confédération. 
Les raisons imputables au monde industriel concernent surtout les sociétés ayant mis en place le dispositif REF pour assurer leur propre relève professionnelle. Par exemple, certaines entreprises publiques sont obligées de former le futur personnel qualifié pour le bien commun public (transports publics, soins). Ainsi, des maisons de retraites publiques ont dû proposer quelques places d'apprentissage au " réseau intégration ", afin de former les assistants/tes socio-éducatifs/ves. Dans le "réseau des transports publics ", le rôle de l'entreprise fédérale est de permettre aux autres entreprises du secteur d'avoir accès aux multiples possibilités de formation ; elle est donc dans l'obligation de participer au réseau. Grâce à la participation au modèle en réseau, on établit un compromis entre deux formes de convention : l'industrielle et la civique. Les raisons évoquées par les entreprises dans le " réseau région" vont alors dans le même sens ; elles souhaitent proposer des places d'apprentissage aux jeunes dans la région afin de contrer la fuite du (futur) personnel qualifié.

\section{2 ... ou améliorer l'employabilité, attirer et insérer plus de jeunes grâce à une formation réputée}

Les entreprises évoquent aussi d'autres motifs à leur engagement dans la formation en réseau. Ces motifs relèvent en fait d'autres conventions qui ont généré, au sein du réseau, des exigences hétérogènes en matière de coordination et qui sont liées par des compromis aux deux raisons principales.

Pour justifier la formation en réseau, quelques entreprises issues du « réseau des transports publics " et du " réseau de l'expédition » évoquent des exigences spécifiques à leur secteur d'activité qui s'inscrivent dans la logique d'action du monde par projets. En effet, diverses entreprises des transports collaborent déjà, la mobilité des personnes et des biens n'étant plus un champ d'action régional mais national ou international. Une PME (petite et moyennes entreprise) ne couvre plus qu'une étape du transport. Le personnel doit être capable de planifier les tâches et d'agir bien au-delà du cadre de sa propre entreprise. D'autres entreprises évoquent le quotidien peu routinier de leur secteur d'activité. Pour justifier le principe de rotation, certaines entreprises avancent que ces expériences sont importantes pour les apprentis; plus tard, en effet, ils devront s'adapter à des situations en constante mutation, caractéristiques du quotidien professionnel actuel.

Certaines entreprises engagées principalement dans le dispositif afin d'intégrer les apprentis, évoquent aussi un monde du travail en mutation. Elles insistent cependant davantage sur le potentiel du modèle en réseau qui permet, grâce à la rotation entre les entreprises, d'acquérir des compétences clés comme la flexibilité, la mobilité, l'indépendance et la capacité de communiquer, compétences qui permettent de faire face à une vie professionnelle de plus en plus complexe (Leemann et Sagelsdorff, 2014). Pour ces entreprises, ces compéteces sont une condition de "l'employabilité à vie ».

La baisse du nombre d'élèves ces dernières années, due aux évolutions démographiques, mais aussi la valorisation sociale de la voie scolaire-académique font craindre aux entre- 
prises de ne plus trouver assez de jeunes motivés et disposant d'un bon niveau scolaire pour la formation professionnelle initiale. C'est pourquoi certaines entreprises souhaitent, grâce au REF, proposer une formation variée afin d'attirer des jeunes. Cet argument visant à augmenter le potentiel de recrutement grâce à une formation attractive repose sur la convention inspirée.

D'autres entreprises misent au contraire sur la réputation du réseau pour attirer des jeunes qualifiés en fin de scolarité.

D'un côté, l'organisation principale véhicule une image qui se veut " moderne et jeune ", contrairement aux entreprises des transports ferroviaires et des transports en bus qui renvoient plutôt une image d'entreprises "vieilles et poussiéreuses ". La mutualisation des ressources permet aussi à l'organisation principale d'être mieux outillée pour promouvoir les formations offertes.

D’un autre côté, ce sont principalement les petites entreprises, celles qui sont les moins connues dans le réseau, qui bénéficient le plus des "marques fortes ", c'est-à-dire des entreprises connues du public et ayant une bonne réputation. Ces dernières rayonnent sur les autres et attirent les candidats comme un " aimant ». Pour les apprentis, elles sont vraisemblablement le " point culminant " de leur formation. Tous ces arguments relèvent du monde de l'opinion.

Les entreprises qui sont tenues d'intégrer des jeunes souhaitent, elles-aussi, grâce à la rotation, leur offrir une formation passionnante et diversifiée (convention inspirée) afin de prévenir les ruptures de contrat en cours de formation.

Rares sont les entreprises qui justifient leur participation par le moindre coût salarial des apprentis par rapport au personnel formé. Le modèle en réseau permet, en outre, une utilisation flexible de ce personnel bon marché, les petits réseaux permettant aux entreprises de s'engager pour un an seulement. Le nombre d'apprentis peut être ajusté en fonction du niveau des commandes. Cette argumentation s'inscrit dans les logiques d'action du monde marchand correspondant au motif de la production. Néanmoins, au regard des résultats de l'enquête, ce motif est relativement isolé.

\section{Une pluralité de motifs de participation, source de conflits}

Comme la présentation des différents motifs de participation au réseau le révèle, le principe du réseau comprend une pluralité de logiques d'action. En REF, elles se sont condensées en un objet de compromis où persistent néanmoins des tensions qui le rendent fragile et instable (Boltanski et Thévenot, 1999, p. 375). L'origine d'un réseau est empreinte de cette pluralité. Si le réseau est à l'initiative d'acteurs économiques, comme c'est le cas du " réseau des transports publics » et du "réseau de l'expédition », alors les logiques d'actions 
qui dominent le compromis se rattachent au monde industriel et se traduisent par un fort engagement de l'organisation principale dans la formation technique. En revanche, si des acteurs gouvernementaux sont à l'origine du réseau, comme c'est le cas pour le "réseau région » et le "réseau intégration », les logiques du REF comme objet de compromis renvoient alors plutôt à une justification du monde civique avec une forte mobilisation de l'organisation principale visant à intégrer les jeunes.

Les compromis sont des solutions provisoires et instables. Les différents motifs d'une participation à la mutualisation de la formation peuvent donc être sources de conflits et de tensions. Ceux-ci s'observent de manière empirique à travers les critiques émises par les entreprises participant au modèle en réseau. Ces critiques visent les services offerts par l'organisation principale, les entreprises exigeant des aménagements et menaçant même de quitter le réseau. On peut les résumer d'un point de vue théorique, en affirmant qu'elles sont émises sous l'angle d'une certaine convention, en opposition à une autre présente dans le modèle en réseau (Thévenot, 2002, p. 184). Nous présentons ci-après les principaux traits des conflits liés au recrutement des apprentis, au contrôle en cours de formation et à l'embauche des apprentis diplômés.

\subsection{Le recrutement et la sélection échappent à l'entreprise}

Lorganisation principale prend en charge le travail, souvent long et complexe, de recrutement et de sélection et effectue cette tâche de façon plus professionnelle que ne pourrait le faire une PME. Mais, en déléguant cette tâche, l'entreprise subit une perte d'influence en matière de sélection des apprentis car ces derniers leur sont simplement attribués.

Des critiques envers ce recrutement organisé selon les principes de la convention industrielle s'appuient sur la convention domestique et de l'opinion. Les deux critiques portent sur le fait que les jeunes intéressés ne peuvent postuler qu'auprès de l'organisation principale et pas directement auprès des entreprises elles-mêmes. L'organisation principale serait impersonnelle et de nombreuses personnes s'y perdraient. Les contacts personnels, comme par exemple le père qui travaille dans l'entreprise, n'auraient plus de pertinence (monde domestique). Certaines entreprises font aussi valoir que les jeunes souhaitent aller dans une entreprise en particulier parce que le nom de la société est connu (monde de l'opinion).

Les entreprises désireuses de participer à la sélection s'appuient sur les principes de la convention domestique. Elles souhaitent regagner en autorité en participant à la définition des critères de sélection, aux entretiens, en testant les jeunes au cours de journées d'essai et en pouvant refuser un jeune lors du placement dans le système de rotation. Cela leur permettrait d'évaluer la compatibilité entre le jeune et l'entreprise et de choisir la personne adéquate pour le métier et le secteur d'activité.

Certaines entreprises critiquent la " qualité » des jeunes. Elles dénoncent le fait que l'organisation principale ne parvient pas à gagner la confiance d'apprentis assez dotés scolaire- 
ment et suffisamment motivés. Une logique marchande est à l'origine de cette critique. En effet, l'organisation principale est rémunérée par les entreprises et elles ne sont pas satisfaites de la qualité de la "matière première ". Cela génère un surcroît de travail au niveau de l'accompagnement des jeunes et le rendement économique est plus faible étant donné la moindre productivité des apprentis.

Pour d'autres entreprises, les problèmes de recrutement sont liés à l'image de l'entreprise renvoyée par le "réseau intégration » et le "réseau région"; selon elles, ces réseaux se centreraient trop vers l'intégration des jeunes socialement défavorisés. Sur fond de la convention de l'opinion, elles critiquent le principe civique de la politique de recrutement des organisations principales qui, selon elles, essaient de donner une chance à chacun.

\subsection{L'entreprise, entre perte d'autorité et baisse de productivité}

Les apprentis sont employés par l'organisation principale. Cette dernière est responsable de la formation et de l'accompagnement. Les principes de rotation et d'accompagnement partagé sont les deux piliers de ce nouveau mode d'apprentissage. Dans ces domaines aussi, l'entreprise perd son influence et le contrôle de la situation, ce qui engendre toujours tensions et mécontentements.

Le temps relativement court (de 6 à 12 mois) passé dans une entreprise ne satisfait pas certaines entreprises; elles le jugent insuffisant pour permettre aux jeunes de se familiariser avec la culture de l'entreprise, les processus et les hiérarchies internes. Lors de l'apprentissage en réseau, il est impossible pour une entreprise de s'engager et d'imposer sa vision et ses objectifs en matière d'éducation et de socialisation des apprentis. Cette critique est issue de la convention domestique et vise la convention par projets du réseau. Elle se retrouve aussi dans les objections concernant l'accompagnement partagé. Les entreprises regrettent le faible pouvoir de décision des formateurs pratiques. Elles dénoncent le fait de devoir faire appel au responsable de formation de l'organisation principale en cas de problème et de ne pas avoir le droit d'émettre des avertissements, par exemple. Cela affaiblit fortement leur autorité.

D'autres entreprises ont aussi critiqué le peu de temps passé dans une entreprise lié au système de rotation (élément du monde par projets). Les arguments utilisés s'appuient sur le principe de la convention marchande. Comme indiqué précédemment, les apprentis restent néanmoins une main-d'œuvre dont le salaire et l'investissement dans l'accompagnement doivent être amortis. Quelques rares entreprises déclarent ouvertement que l'apprenti doit les soulager, par exemple en palliant les absences des employés durant la semaine ou les congés. Le changement fréquent d'entreprises fait perdre à l'apprenti le savoir acquis et empêche que les objectifs de rendement et de réduction de charge de travail soient atteints. 


\subsection{La concurrence fait obstacle à l'emploi d'apprentis diplômés}

L'objectif principal du REF, constituer à long terme la relève professionnelle, est paradoxalement menacé par le principe de rotation. Comme les apprentis effectuent leur apprentissage dans plusieurs entreprises, chacune d'elles a la possibilité d'utiliser des moyens adaptés pour tisser des liens avec les jeunes au cours de la formation, dans l'objectif de les embaucher à l'issue de l'apprentissage. Les entreprises deviennent ainsi concurrentes. Cette problématique est présente dans le "réseau des transports publics » et le "réseau de l'expédition ». L'entreprise fédérale appartenant au "réseau des transports publics ", ayant de forts besoins en main-d'œuvre, est relativement insatisfaite du nombre d'apprentis qu'elle a pu embaucher. Dans le système de rotation, elle dénonce le fait qu'un séjour de courte durée dans l'entreprise (6 mois) ne permette pas de donner au jeune l'impression d'appartenir à celle-ci, ni la possibilité de s'identifier à elle, ce qui nuit à son attractivité. Elle critique ainsi le principe marchand qui intervient particulièrement lors du recrutement du personnel qualifié issu des rotations interentreprises. Cette critique s'inscrit dans des logiques propres au monde industriel. Dans la perception de cette entreprise fédérale, les entreprises de l'aviation et celles situées dans des zones de montagnes ont de meilleures chances sur le marché car leurs champs d'activité sont plus attractifs : "L'aéroport » synonyme de vacances, de pays lointains et d'aventure ; les " chemins de fer dans l'Oberland " qui évoquent les sports, les loisirs, et les magnifiques paysages de montagne...

\section{Conclusion}

Au final, deux motifs ressortent dans la participation à une formation coopérative du réseau d'entreprises formatrices (REF).

D'un côté, celui du monde industriel qui repose sur une logique d'action, d'efficacité, d'expertise, et de maîtrise : former une relève de personnel qualifié à long terme grâce au REF qui fournit un aperçu de divers champs d'activité professionnelle, et grâce à l'expertise de l'entreprise principale.

De l'autre, le motif relevant du monde civique où la solidarité, l'égalité et l'intégration sociale font partie des ordres de justification : intégrer des jeunes dans le monde du travail par la création de places d'apprentissage supplémentaires dans les REF et par un recrutement, une formation et un accompagnement professionnel pris en charge par l'organisation principale.

D'autres motifs reposent sur le monde de justification par projets (acquisition de compétences clés grâce au système de rotation), sur le monde inspiré (obtention d'apprentis qualifiés grâce à une formation variée), le monde de l'opinion (obtention d'apprentis qualifiés grâce à la présence d'entreprises connues dans le réseau) ou le monde marchand (apprentis comme main-d'œuvre bon marché). 
Selon l'origine d'un réseau, fondé par des acteurs économiques ou par des acteurs gouvernementaux, dans un REF se mêlent différents motifs de participation. Des compromis permettent de répondre à ces exigences de coordination hétérogènes et de stabiliser temporairement la situation.

Cependant, ces compromis ont pour conséquence de faire naître des conflits en série tout au long des étapes de la formation : le recrutement et la sélection, la rotation, la formation et l'accompagnement, l'intégration des apprentis diplômés dans le monde du travail.

Les acteurs issus des entreprises émettent des critiques vis-à-vis de ce modèle de formation - notamment le peu d'influence dont disposent les entreprises, le faible attachement des apprentis à l'entreprise ou le travail supplémentaire nécessaire à l'initiation des apprentis, de même que le faible rendement des apprentis comme main-d'œuvre. Ces critiques s'appuient sur un monde pour dénoncer la logique d'action d'un autre monde inscrit dans le réseau.

Aussi, l'organisation principale du REF va devoir sans cesse chercher un équilibre entre les diverses attentes, souvent contraires, des entreprises au sein du réseau. Elle prend en compte les souhaits des entreprises, surtout si celles-ci sont très importantes pour la pérennité du réseau. Elle cherche de nouveaux compromis, notamment quand la pérennité de l'organisation dépend de la réussite du modèle. Les REF sont donc organisés de manière complexe et instable, les compromis et les conflits s'y succèdent (Leemann et Imdorf, $2015 \mathrm{a} ; 2015 \mathrm{~b})$. Cela peut expliquer pourquoi ce modèle prometteur de formation professionnelle peine au final à s'imposer.

Afin de développer les réseaux d'apprentissage présentés ici, nous pouvons formuler quelques préconisations (Leemann et Imdorf, 2015b) :

Il conviendrait, en effet, d'assurer le principe de rotation entre entreprises - point fort de ce dispositif d'apprentissage - en accordant plus d'initiatives aux entreprises en matière de recrutement, de placement dans le système de rotation et d'encadrement par les formateurs pratiques. L'organisation principale pourrait, d'une part, associer les entreprises du réseau à la définition des critères de sélection et aux entretiens, et proposer des journées d'essai dans les entreprises avant que les apprentis ne soient placés ; d'autre part, elle pourrait accorder aux entreprises plus de pouvoir de contrôle sur les apprentis.

Toutes les entreprises doivent pouvoir disposer d'une main-d'œuvre d'apprentis; en outre, l'investissement en matière d'accompagnement, de placement des apprentis et de coûts annuels supportés par les entreprises doit être réparti équitablement, en tenant compte de la durée totale de l'apprentissage (soit trois à quatre ans en Suisse).

En outre, l'organisation principale doit s'assurer (en lien avec l'association professionnelle) que l'embauche des apprentis diplômés s'effectue en toute transparence - par exemple en institutionnalisant un " jour d'embauche " permettant aux entreprises et aux apprentis 
futurs diplômés de mieux se connaître - et offrir les mêmes salaires aux apprentis diplômés en début de carrière.

Pour développer ces réseaux d'apprentissage, il serait judicueux de mobiliser une comparaison internationale avec des pays où la formation en réseaux est répandue. Ainsi, en Norvège, la plupart des apprentis se voient proposer une place d'apprentissage via des agences intermédiaires locales (local training agencies) qui coopèrent avec les entreprises formatrices mais sans avoir recours au système de rotation (Høst, 2008). Ces agences reçoivent de fortes subventions de l'Etat pour coordonner la formation professionnelle sur un plan local, ce qui peut favoriser le développement de ce dispositif.

\section{Bibliographie}

BMBF (Bundesministerium für Bildung und Forschung) (2011), Verbundausbildung die Ausbildungsform der Zukunft?, Jobstarter Praxis, Bd 6, Bonn, BIBB.

Boltanski L. \& Chiapello E. (1999), Le nouvel esprit du capitalisme, Paris, Gallimard.

Boltanski L. \& Thévenot L. (1991), De la justification. Les économies de la grandeur, Paris, Gallimard.

Boltanski L. \& Thévenot L. (1999), “The Sociology of Critical Capacity”, European Journal of Social Theory 2(3), pp. 359-377.

Diaz-Bone R. \& Thévenot L. (2010), "Sociologie des conventions », Trivium n ${ }^{\circ}$. URL: http:// trivium.revues.org/3626. Consulté le 21 janvier 2015.

Diaz-Bone R. (2011), "The Methodological Standpoint of the "économie des conventions' ", Historical Social Research 36(4), pp. 43-63.

Drinkhut V. \& Schlottau W. (2003), « Förderung von Ausbildungsverbünden: Anschub zu mehr und besseren Ausbildungsplätzen? in Verbundausbildung - Organisationsformen, Förderung, Praxisbeispiele, Rechtsfragen. Ergebnisse, par BIBB, Bonn, BIBB, pp. 2-37.

Eymard-Duvernay F. (2008), "Justesse et justice dans les recrutements ", Formation Emploi, n 101, pp. 55-69.

Hoeckel K., Field S., Grubb W. N. (2009) “Learning for Jobs”, OECD Reviews of Vocational Education and Training Switzerland, OECD.

Høst H. (2008), Continuity and Change in Norwegian Vocational Education and Training (VET), Oslo: NIFU STEP.

Imdorf, C. Leemann R. J. (2012), "New models of apprenticeship and equal employment opportunity. Do training networks enhance fair hiring practices?", Journal of Vocational Education \& Training 64(1), pp. 57-4. 
Imdorf Ch. \& Seiterle N. (2015), « La formation professionnelle dans le cadre des réseaux d'entreprises formatrices comme aide à l'intégration des jeunes issus de l'immigration ", in Les Inégalités Scolaires en Suisse. Ecole, Société et Politiques Educatives, par Felouzis G. et Goastellec G., Berne, Peter Lang, pp .14-160.

Jagd S. (2011), "Pragmatic Sociology and Competing Orders of Worth in Organizations", European Journal of Social Theory 14(3), pp. 343-359.

Leemann R.J. \& Birr L. (2015), „Der Aufbau von Erfahrungswissen in der betrieblichen Ausbildung von Ausbildungsverbünden: Potenziale und Bedingungen ", in Soziale Inwertsetzung von Wissen, Erfahrung und Kompetenz in der Berufsbildung, par Dietzen A., Powell J.J.W., Bahl A. et Lassnigg L., Weinheim und München, Beltz Juventa, pp. 238-259.

Leemann R.J. \& ImdorfCh. (2015a), „Ausbildungsverbünde als Organisationsnetzwerke“, in Konventionen und Organisationen, par Knoll L, Wiesbaden, Springer VS, pp. 13-161.

Leemann R.J. \& Imdorf Ch. (2015b) “Cooperative VET in Training Networks: Analysing the Free-Rider Problem in a Sociology-of-Conventions Perspective”, International Journal for Research in Vocational Education and Training (IJRVET) 2(4), pp. 184-207.

Leemann R.J. \& Sagelsdorff R. (2014), „Der Erwerb von Schlüsselkompetenzen in Ausbildungsverbünden “, Sozialer Fortschritt 63(4-5), pp. 107-117.

OFFT (Office fédéral de la formation professionnelle et de la technologie) (2008), Résultats Evaluation des réseaux d'entreprises formatrices, Berne, OFFT.

Schönfeld G., Wenzelmann F., Dionisius R., Pfeifer H., Walden G. (2010), Kosten und Nutzen der dualen Ausbildung aus Sicht der Betriebe. Ergebnisse der vierten BIBB-KostenNutzen-Erhebung, Bielefeld, Bertelsmann.

Strupler M. \& Wolter St. C. (2012), Die duale Lehre : eine Erfolgsgeschichte - auch für Betriebe, Glarus/Chur, Rüegger.

Thévenot L. (2002), "Conventions of co-ordination and the framing of uncertainty", in Intersubjectivity in Economics, par Fullbrook E., London, Routledge, pp. 181-197.

Walther B. \& Renold U. (2005), „Lehrbetriebsverbund - neue Chancen für Klein- und Mittelbetriebe", Die Volkswirtschaft, 4, pp. 39-42.

Wenzelmann F. (2012), „Ausbildungsmotive und die Zeitaufteilung der Auszubildenden im Betrieb “, Journal of Labour Market Research, 45, pp. 125-145.

Wenzelmann F., Schönfeld G., Pfeifer H., Dionisius R. (2009), Betriebliche Berufsausbildung: Eine lohnende Investition für Betriebe, BIBB-Report Heft 8, Bonn, BIBB.

Yin R.K. (2009), Case Study Research. Design and Methods, Thousand Oaks, Sage. 